\title{
MENSURANDO OS IMPACTOS DIRETOS E INDIRETOS DO CAPITAL HUMANO SOBRE O CRESCIMENTO*
}

Luciano Nakabashi ${ }^{\S}$

Lízia de Figueiredo

\begin{abstract}
RESUMO
O objetivo do presente estudo é o de avaliar os diferentes canais pelos quais o capital humano afeta o nível e a taxa de crescimento da renda por trabalhador. A análise empírica é baseada em um modelo que incorpora canais em que a primeira variável afeta a segunda via: 1) melhora na produtividade marginal do trabalho;2) criação de tecnologia, e 3) difusão de tecnologia. A consideração de variados canais em que o capital humano afeta a taxa de crescimento da renda se deve à complexidade da relação entre estas. Assim, caso ocorra a omissão de alguns canais, podemos estar incorrendo em erros de especificação do modelo e obter coeficientes tendenciosos.
\end{abstract}

Palavras-chave: capital humano, criação de tecnologia, difusão de tecnologia.

\begin{abstract}
The objective of this study is to evaluate the different channels wherein human capital affects income per worker level and growth. The empirical analysis is based in a model that incorporates several channels in which human capital affects the rate of income per worker growth: 1) improving the marginal productivity of labor; 2) through creation of technology; and 3) diffusion of technology. The consideration of several channels wherein human capital affects income is due to the complexity of the relationship between these two variables. Therefore, if we rule out any channel we can incur in model specification errors.
\end{abstract}

Keywords: human capital, creation of technology, diffusion of technology.

JEL classification: C23, I21, O11, O33.

* Os autores gostariam de agradecer os valiosos comentários de Márcio Salvato, Gabriel Porcile, Maurício Bittencourt, Fábio Gomes, Marcos Flávio da Cunha Resende e de dois pareceristas anônimos.

$\S$ Doutor em Economia pelo Cedeplar/UFMG, professor adjunto da UFPR e coordenador do boletim de Economia \& Tecnolgia; uma publicação do Centro de Estudos Econômicos (CEPEC/UFPR). Endereço para contato: Av. Prefeito Lothário Meissner, 632 - térreo. Departamento de Economia, Bairro Jardim Botànico. CEP: 80210-170, Curitiba - PR. E-mail: luciano.nakabashi@ufpr.br.

a Doutora em Economia pela University of Nottinghan, professora adjunta do CEDEPLAR/UFMG e Coordenadora do EXAME-ANPEC.

Recebido em junho de 2006. Aceito para publicação em março de 2008. 


\section{INTRODUÇÃo}

Existe uma ampla discussão sobre o papel do capital humano no crescimento das economias e ela está longe de ser finalizada. Muitos pesquisadores acreditam que o papel desse fator sobre o crescimento é essencial. Schultz $(1960,1961,1962)$ foi um dos principais economistas a enfatizar as relações macroeconômicas entre capital humano e crescimento econômico:

The hypothesis here advanced is that the inclusion of human capital will show that the ratio of all capital to income is not declining. Producer goods-structures, equipment and inventories - a particular stock of capital has been declining relative to income. Meanwhile, however, the stock of human capital has been rising relative to income. If the ratio of all capital to income remains essentially constant, then the unexplained economic growth which has been so puzzling originates mainly out of the rise in the stock of human capital. (Schultz,1962, p. 1).

Para Schultz, a inclusão da acumulação de capital humano é um elemento-chave na compreensão do processo de crescimento econômico no longo prazo, sendo sua principal fonte. Desse modo, Schultz fornece a base teórica para o desenvolvimento dos modelos de crescimento endógeno da segunda metade dos anos 1980, como o de Romer (1986), por exemplo.

Apesar de a teoria enfatizar o papel do capital humano sobre o crescimento e desenvolvimento das nações, há vários estudos macroeconômicos que não encontram evidências empíricas de que tal fator seja importante, com um destaque especial para o estudo realizado por Pritchett (2001). Outros estudos tentam encontrar explicação para a inexistência da correlação entre crescimento e capital humano.

Por outro lado, existe uma grande quantidade de estudos microeconômicos empíricos que dá suporte à visão de que o capital humano é um elemento crucial para o crescimento da renda de uma forma direta. Muitos estudos empíricos apontam que os retornos dos investimentos em capital humano são elevados tanto em países em desenvolvimento quanto nos desenvolvidos (Dowrick, 2003).

Alguns possíveis motivos para essa divergência entre os resultados dos estudos micro e macroeconômicos são os erros de especificação de modelo e os dados de baixa qualidade. Temple (1999) mostra, ainda, que outliers influentes podem levar a distorções dos resultados. Se essas observações são omitidas, usando o método least trimmed squares, a conclusão é que o fator capital humano é mais importante do que realmente aparenta ser.

Um exemplo de análise que incorpora a variável qualidade do capital humano é o estudo de Hanushek e Kimko (2000). De fato, os resultados encontrados indicam uma forte relação entre qualidade da educação e crescimento da renda per capita. Barro e Lee (2001) também encontram uma relação positiva entre qualidade e taxas de crescimento da renda real per capita em dados de corte. Eles empregam os resultados dos testes TIMMS $^{1}$ para estudantes e IALS ${ }^{2}$ para adultos, como proxy para qualidade do capital humano. Para o caso brasileiro, temos o estudo realizado por Nakabashi e Salvato (2007), onde os resultados são semelhantes, ou seja, a qualidade educacional é fundamental na determinação da renda e da sua taxa de crescimento.

1 The Third International Mathematics and Science Study in 1994 and 1995.

2 International Adult Literacy Survey. 
Alguns exemplos de estudos empíricos que testam diferentes especificações de modelos e formas funcionais são aqueles feitos por Benhabib e Spiegel (1994, 2002) e Islam (1995). Os resultados dão suporte ao modelo teórico de Nelson e Phelps (1966), em que o capital humano é um importante fator na determinação do crescimento/nível de renda por ser um facilitador do processo de difusão tecnológica.

Teles (2005) mostra ainda evidências de que a dinâmica do capital humano sobre o crescimento da renda pode ser diferente, quando considerados grupos distintos de países de acordo com o nível de renda. Para o grupo de países mais ricos, o modelo de Lucas (1988) seria o mais apropriado, enquanto que, para os países de renda mais baixa, o mais adequado seria o de Nelson e Phelps (1966).

Tendo essa discussão como pano de fundo, o objetivo do presente estudo é analisar os diferentes impactos do capital humano na determinação da taxa de crescimento da renda, ou seja, seus efeitos diretos e indiretos. Por efeitos diretos do capital humano, nós nos referimos àqueles que afetam a renda por meio da melhora na produtividade marginal do trabalho, mantendo todos os outros fatores constantes (capital e tecnologia), isto é, da maior habilidade dos trabalhadores na realização de suas respectivas tarefas. Ele é representado pela introdução do capital humano de forma direta na função de produção. Os efeitos indiretos são aqueles que afetam a quantidade de tecnologia disponível para ser utilizada no processo de produção. Assim, são os fatores que influenciam a criação e difusão de tecnologia.

A introdução desses vários canais tem como objetivo a utilização de um modelo mais completo para evitar erros de especificação, pois, no caso de omissão de uma variável relevante, que esteja correlacionada com pelo menos uma variável explicativa, obtemos estimadores dos parâmetros tendenciosos e inconsistentes. Portanto, a grande contribuição do trabalho é testar uma especificação mais complexa dos fatores que afetam a taxa de crescimento econômico, sem perder de vista a importância da formalização na formulação das equações a serem estimadas empiricamente. A importância da formalização é devida ao maior suporte teórico em relação às variáveis relevantes no processo e na forma funcional a ser empregada, ao invés de se utilizar variáveis de maneira ad hoc.

Portanto, o foco do trabalho é na especificação do modelo a ser estimado, embora a consideração da qualidade desse fator seja de extrema importância. Essa limitação do tema se justifica pela sua complexidade.

$\mathrm{Na}$ análise empírica serão empregadas duas amostras distintas. Uma delas é composta por 96 países em quatro períodos de tempo (1985, 1990, 1995, 2000), mas a quantidade de observações não se iguala a 384, pois alguns países não possuem dados para todo o período. A outra amostra é composta por 29 países com uma base de dados mais completa e de maior confiabilidade, ${ }^{3}$ considerando o mesmo período da amostra anterior.

Além dessa introdução, o presente trabalho é composto pela Seção 2, onde se apresenta o modelo utilizado na análise empírica. A Seção 3 apresenta a metodologia e os dados. Finalmente, na quarta seção, são apresentados e discutidos os resultados.

3 Assim, as regressões são feitas utilizando dados de painel com dados incompletos (não balanceado). 


\section{O MODELO}

A função de produção é a seguinte:

$$
Y_{i t}=K_{i t}^{\alpha} H_{i t}^{\beta}(A L)_{i t}^{1-\alpha-\beta}
$$

em que $Y$ é o nível de renda, $K$ é o nível de capital físico, $H$ é o nível de capital humano, $A$ é o nível de tecnologia e $L$ é a quantidade do fator trabalho usado no processo de produção. Os subscritos $i$ e $t$ se referem ao país $i$ no tempo $t$. Adicionalmente, $\alpha, \beta$, e $1-\alpha-\beta$ são as parcelas de cada um dos fatores na renda. Derivando a equação (1) em relação ao tempo, temos:

$$
\frac{\dot{Y}_{i t}}{Y_{i t}}=\alpha \frac{\dot{K}_{i t}}{K_{i t}}+\beta \frac{\dot{H}_{i t}}{H_{i t}}+(1-\alpha-\beta) \frac{\dot{A}_{i t}}{A_{i t}}+(1-\alpha-\beta) \frac{\dot{L}_{i t}}{L_{i t}}
$$

em que $\dot{X}$ corresponde a $\partial X / \partial t$ e $X$ representa qualquer uma das variáveis na equação (2). Dividindo e multiplicando o lado esquerdo e os dois primeiros termos da equação (2) por $L$, produz:

$$
\frac{\dot{Y}_{i t} / L_{i t}}{Y_{i t} / L_{i t}}=\alpha \frac{\dot{K}_{i t} / L_{i t}}{K_{i t} / L_{i t}}+\beta \frac{\dot{H}_{i t} / L_{i t}}{H_{i t} / L_{i t}}+(1-\alpha-\beta) \frac{\dot{A}_{i t}}{A_{i t}}+(1-\alpha-\beta) \frac{\dot{L}_{i t}}{L_{i t}}
$$

Pela validade de (4),

$$
\frac{\dot{X}}{L}=\dot{x}+n x
$$

em que $X$ representa qualquer uma das variáveis acima, $x=X / L$, e $n=\dot{L} / L$, a equação (3) pode ser rescrita como

$$
\frac{\dot{y_{i t}}}{y_{i t}}=\alpha \frac{\dot{k_{i t}}}{k_{i t}}+\beta \frac{\dot{h_{i t}}}{h_{i t}}+(1-\alpha-\beta) \frac{\dot{A_{i t}}}{A_{i t}}
$$

Fazendo as mesmas suposições do modelo ampliado de Solow sobre as equações que regem a dinâmica da acumulação dos fatores capitais físico e humano, temos:

$$
\begin{aligned}
& \dot{K}_{i t}=s_{i t}^{k} Y_{i t}-\delta K_{i t} \\
& \dot{H}_{i t}=s_{i t}^{h} Y_{i t}-\delta H_{i t}
\end{aligned}
$$

onde $s_{i t}^{k}$ e $s_{i t}^{h}$ são as respectivas taxas de investimento (que é igual às taxas de poupança) em capital físico e humano para o país $i$ no período $t$ e $\delta$ é a taxa de depreciação de cada um dos tipos de capital que, por suposição, são iguais. Se as duas equações acima forem expressas na forma de 
crescimento do capital por unidades efetivas de trabalho, encontramos:

$$
\begin{aligned}
& \dot{k}_{i t}=s_{i t}^{k} y_{i t}-\left(n_{i t}+\delta\right) k_{i t} \\
& \dot{h}_{i t}=s_{i t}^{h} y_{i t}-\left(n_{i t}+\delta\right) h_{i t}
\end{aligned}
$$

O primeiro termo do lado direito das equações acima representa o investimento em capital por unidades de trabalho. Portanto, elas podem ser representadas por:

$$
\begin{aligned}
& \frac{\dot{k_{i t}}}{k_{i t}}=i_{i t}^{k}-\left(n_{i t}+\delta\right) \\
& \frac{\dot{h_{i t}}}{h_{i t}}=i_{i t}^{h}-\left(n_{i t}+\delta\right)
\end{aligned}
$$

em que $i^{k}$ e $i^{h}$ representam a razão entre os investimentos em capitais físico e humano em unidades de trabalho e as quantidades de capitais físico e humano por unidade de trabalho $\left[i^{k}=\left(s^{k} y\right) / k\right.$ e $i^{h}$ $\left.=\left(s^{h} y\right) / h\right] \cdot{ }^{4}$ Fazendo o uso de (10) e (11) na equação (5), temos:

$$
\frac{\dot{y_{i t}}}{y_{i t}}=\alpha i_{i t}^{k}+\beta i_{i t}^{h}+(1-\alpha-\beta) \frac{\dot{A}_{i t}}{A_{i t}}-(\alpha+\beta)\left(n_{i t}+\delta\right)
$$

Se utilizarmos a especificação de Benhabib e Spiegel (1994), que é baseada em Nelson e Phelps (1996) e Romer (1990), a dinâmica do progresso tecnológico pode ser representada por:

$$
\dot{A}_{i t}=\pi H_{A i t} A_{i t}^{\xi}+\varphi h_{i t}\left(T_{t}-A_{i t}\right)+\varepsilon
$$

em que $H_{A}$ é a quantidade de capital humano empregada em pesquisa e desenvolvimento (P\&D), $T_{t}$ é a fronteira tecnológica no tempo $t, \xi \leq 1$ relaxa a suposição knife-edge, ${ }^{5}$ permitindo que esse canal, por meio do qual o capital humano afeta o avanço tecnológico, não gere, necessariamente, crescimento endógeno (Solow, 1994), e $\varepsilon$ é o termo de erro aleatório que representa outros fatores que podem afetar o crescimento do nível de tecnologia. Usando (13) em (12):

4 Pela dificuldade em se mensurar o estoque de capital físico em cada país, a proxy para $i_{i t}^{k}$ utilizada na análise empírica é o investimento por trabalhador. Para manter o mesmo padrão, a proxy para $i_{i t}^{h}$ é a variação de anos de escola da população com 25 anos ou mais. Se emprega a população com mais de 25 como uma proxy dos trabalhadores da economia.

5 Knife Edge Assumption é a suposição de que $\xi=1$. Isso implica, no presente modelo, que a quantidade de capital humano destinada à pesquisa e desenvolvimento é capaz de gerar crescimento endógeno constante, como se pode ver pela equação (14). Caso $\xi<1$, o avanço tecnológico dificulta o surgimento de inovações, restringindo o crescimento no estado estacionário. Caso $\xi>1$, o crescimento seria explosivo com o avanço tecnológico. 


$$
\begin{aligned}
& \frac{\dot{y}_{i t}}{y_{i t}}=\alpha i_{i t}^{k}+\beta i_{i t}^{h}+\frac{(1-\alpha-\beta) \pi H_{A i t}}{A_{i t}^{1-\xi}}+(1-\alpha-\beta) \varphi h_{i t}\left(\frac{T_{t}-A_{i t}}{A_{i t}}\right)-(\alpha+\beta)\left(n_{i t}+\delta\right) \\
& +\frac{(1-\alpha-\beta)}{A_{i t}} \varepsilon
\end{aligned}
$$

Pela equação (14), o capital humano afeta a taxa de crescimento da renda por unidade de trabalho por três vias distintas: 1) diretamente, onde o investimento em capital humano mensura a mudança nas habilidades dos trabalhadores que estão empregados no processo de produção; 2) indiretamente via criação de tecnologia, onde o que importa é a quantidade de capital humano empregada em atividades de $\mathrm{P} \& \mathrm{D}$ e a quantidade de tecnologia previamente acumulada; e 3) indiretamente pela facilitação do emprego de tecnologia - criada previamente em outras regiões/países - no processo de produção (difusão de tecnologia). ${ }^{6}$

Entretanto, o processo de difusão de tecnologia é bem mais complexo do que aquele expresso por (13), ou seja, o capital humano não é o único fator que influencia no processo de difusão. Seguindo Coe e Helpman (1995); Coe, Helpman e Hoffmaister (1997); Keller (1999), e Connolly (2003), o nível de importações também pode ser um canal essencial no processo de difusão de tecnologia. Outros estudos mostram que os Investimentos Estrangeiros Diretos (IED) são fundamentais nesse processo, como Javorcik (2004), Xu (2000) e Borensztein et al. (1998). Além disso, alguns estudos sugerem ainda que o capital humano interage com os dois canais acima mencionados de modo a intensificar o processo de difusão.

Desse modo, um modelo mais geral deveria incluir não apenas o papel do capital humano no processo de difusão, mas também das importações e do nível de IED. A introdução desses elementos na análise transforma a equação (13) em:

$$
\dot{A}_{i t}=\pi H_{A i t} A_{i t}^{\xi}+\varphi h_{i t}\left(T_{t}-A_{i t}\right)+\left(\varphi_{2} F D I_{i t}+\varphi_{3} M_{i t}+\varphi_{4} F D I_{i t} h_{i t}+\varphi_{5} M_{i t} h_{i t}\right)\left(T_{t}-A_{i t}\right)+\varepsilon
$$

em que é feita a suposição de que os canais de difusão são mais efetivos quando existe um maior nível de tecnologia que pode ser utilizada pelo país em questão, ou seja, quando ele está distante da fronteira tecnológica. Dividindo $(15)$ por $\mathrm{A}_{\mathrm{it}}$, temos:

$$
\frac{\dot{A}_{i t}}{A_{i t}}=\frac{\pi H_{A i t}}{A_{i t}^{1-\xi}}+\left(\varphi h_{i t}+\varphi_{2} F D I_{i t}+\varphi_{3} M_{i t}+\varphi_{4} F D I_{i t} h_{i t}+\varphi_{5} M_{i t} h\right)\left(\frac{T_{t}-A_{i t}}{A_{i t}}\right)+\frac{\varepsilon}{A_{i t}}
$$

Empregando (16) em (12):

6 As proxies para mensurar as várias maneiras pelo qual o capital humano afeta a taxa de crescimento da renda por trabalhador são a variação de anos de escola da população com 25 anos ou mais como proxy para investimento em capital humano por trabalhador, anos de escola da população acima de 25 como proxy para estoque por trabalhador e publicações científicas/patentes concedidas como proxy para capital humano investindo em criação de tecnologia. 


$$
\begin{aligned}
& \frac{\dot{y}_{i t}}{y_{i t}}=\alpha i_{i t}^{k}+\beta i_{i t}^{h}+(1-\alpha-\beta)\left[\frac{\pi H_{A i t}}{A_{i t}^{1-\xi}}+\left(\varphi h_{i t}+\varphi_{2} F D I_{i t}+\varphi_{3} M_{i t}+\varphi_{4} F D I_{i t} h_{i t}\right.\right. \\
& \left.\left.+\varphi_{5} M_{i t} h_{i t}\right)\left(\frac{T_{t}-A_{i t}}{A_{i t}}\right)\right]-(\alpha+\beta)\left(n_{i t}+\delta\right)+\frac{(1-\alpha-\beta)}{A_{i t}} \varepsilon
\end{aligned}
$$

Com essa especificação, podemos avaliar os impactos diretos e indiretos do capital humano sobre a taxa de crescimento da renda por trabalhador. Nessa equação é importante ressaltar que o crescimento da renda per capita não é, necessariamente, gerado de forma endógena, já que os dois primeiros termos tendem a ter um efeito cada vez menor conforme se elevam os estoques dos capitais físico e humano por trabalhador, pois $i^{k}=\left(s^{k} y\right) / k$ e $i^{h}=\left(s^{h} y\right) / h$. No entanto, isso não acontece quando a razão $Y / K$ e $Y / H$ é não decrescente, sendo possível caso os retornos marginais cruzados entre os dois tipos de capital sejam suficientes para compensar o retorno marginal decrescente de cada um deles em relação à produção, ou seja, quando $\alpha+\beta=1$.

O terceiro elemento depende do valor de $\xi$, gerando crescimento endógeno, quando $\xi=$ 1 (knife-edge assumption). O quarto termo depende da distância entre o nível de tecnologia da fronteira e do país em questão, pois quando os dois são os mesmos, o crescimento da renda por trabalhador proveniente desse canal é nulo. Assim, quando todos os países chegassem na fronteira, eles cresceriam à taxa de crescimento da fronteira tecnológica, $\gamma$. O último termo é apenas o da depreciação do capital.

\section{Metodologia E dAdos}

O período do estudo vai de 1985 a 2000, com dados para cada cinco anos. A primeira amostra é composta por 96 países em quatro períodos distintos (1985, 1990, 1995, 2000), mas o tamanho da amostra não se iguala a 384 pela ausência de dados em alguns períodos. A segunda amostra é composta por 29 países, cujos dados são compostos por uma base mais completa e confiável. ${ }^{8}$ Essa amostra é empregada com fins de comparação dos resultados. Entretanto, como esta é composta por países de renda mais elevada, os resultados também indicam se as variáveis do modelo têm um impacto diferenciado nos países mais ricos em relação à média de uma amostra que é mais representativa de todos os países.

Pela equação (17), podemos ver quais as variáveis necessárias para avaliar os vários canais em que o capital humano afeta o crescimento da renda por trabalhador. ${ }^{9}$ A proxy para investimento em capital humano por trabalhador é a variação dos anos de escola da população acima de 25 anos de Barro e Lee (2001). O estoque de capital humano por trabalhador é mensurado pelos anos de escola da população acima de 25 anos de Barro e Lee (2001). O investimento em capital físico por

7 No entanto, esse caso não faz parte das suposições do modelo, como podemos verificar pela equação (1).

8 Os países que compõem cada amostra estão no Apêndice A.

9 As estatísticas descritivas das variáveis utilizadas se encontram no Apêndice B. 
trabalhador é composto pela taxa de investimento multiplicada pela renda por trabalhador, ambas retiradas da Penn World Tables 6.1, em preços constantes (1996).

Para a maior amostra, a proxy para quantidade de capital humano alocada para P\&D é o número de patentes por habitantes concedidas pelo United States Patent and Trademark Office (USPTO) da Organização de Cooperação e Desenvolvimento Econômico (OCDE). Pelo fato de essa medida ser influenciada pela vantagem que os aplicadores domésticos possuem em relação aos seus congêneres estrangeiros, a proxy para quantidade de capital humano alocada para $\mathrm{P} \& \mathrm{D}$ da menor amostra é o número de publicações científicas por habitante, ${ }^{10}$ que também tem como fonte a OCDE (Science, Technology and Industrial Outlook - 2002).

A base de dados para investimentos estrangeiros diretos é da Conferência das Nações Unidas sobre Comércio e Desenvolvimento (UNCTAD). As importações são das Contas Nacionais da Penn World Tables. Ambas as séries foram divididas pela população acima de 25 anos, de Barro e Lee (2001). A variação da força de trabalho é medida pelo crescimento da população acima de 25 anos e essa variável é utilizada como proxy para depreciação efetiva do capital. ${ }^{11}$ A fonte também é Barro e Lee (2001).

A variável da equação (17), que ainda não foi mencionada, é o nível de tecnologia da fronteira e de cada um dos países em questão. Ela é calculada por meio do emprego das equações de regressão de efeitos fixos baseadas nas regressões em nível, ou seja, de acordo com a seguinte especificação:

$$
\ln \left(y_{i t}^{*}\right)=\eta a_{i}+\frac{\beta}{1-\beta} \ln \left(s_{i t}^{k}\right)+\frac{\alpha}{1-\beta} \ln \left(h_{i t}^{*}\right)-\frac{\beta}{1-\beta} \ln \left(\delta+n_{i t}+g\right)+\varepsilon_{i t}^{\prime}
$$

em que o sobrescrito ${ }^{*}$ denota que a variável se encontra no estado estacionário, $\eta=(1-\alpha$ $-\beta) /(1-\beta), \varepsilon^{\prime}=\eta \varepsilon$ e $a_{i}$ é a variável dummy que representa as especificidades de cada país. ${ }^{12}$ Como citado anteriormente, as variáveis representadas por letras minúsculas estão divididas pela quantidade de trabalho $(L)$.

A proxy para o nível de tecnologia de cada país é o coeficiente da variável dummy de cada um deles $(\eta)$, pois esses coeficientes expressam as especificidades de cada país que não foram capturadas pelas variáveis explicativas. No entanto, pelo fato de as variáveis na equação (18) estarem em $l n$, o nível de tecnologia de cada país é medido pela exponencial do coeficiente das variáveis dummies. Como os coeficientes destas são fixos para cada país, considera-se que o nível de tecnologia de cada um deles é constante através do tempo.

A distância tecnológica de cada país em relação à fronteira é a diferença do maior coeficiente da variável dummy de todos os países (nesse caso dos EUA que, portanto, é a fronteira tecnológica) e o coeficiente da variável dummy do país em questão (país $i$ ), dividida pelo coeficiente da variável dummy do último. Em termos algébricos, a distância da fronteira é:

10 Nós poderíamos usar a triadic patent families: um conjunto de patentes provenientes do European Patent Office (EPO), Japanese Patent Office (JPO) e USPTO. Entretanto, no período de análise, ela está disponível apenas para os anos 1990 e 1995.

11 Utilizamos os crescimento da população acima de 25 anos, pois essa é uma idade em que os indivíduos, geralmente, entram no mercado de trabalho. A utilização dessa proxy como depreciação efetiva do capital $(\mathrm{n}+\delta)$ supõe que as taxas de depreciação $(\delta)$ sejam as mesmas para todos os países.

12 Para derivação da equação (18), ver Nakabashi (2005). 


$$
\frac{T_{t}-A_{i t}}{A_{i t}}
$$

onde a proxy para $\mathrm{T}_{\mathrm{t}}$ é o coeficiente da variável dummy dos EUA e $\mathrm{A}_{\mathrm{it}}$ é o coeficiente da variável dummy do país em questão, com ambas no período t. Assim, a distância dos Estados Unidos em relação à fronteira é zero. Desse modo, todas as suas variáveis apresentadas na equação (17) que envolvam a distância em relação à fronteira são iguais a zero. Isso significa, de acordo com o modelo apresentado, que esse país não pode se aproveitar da difusão tecnológica porque ele já está na fronteira.

As fontes utilizadas para estimar a equação (18) foram as mesmas listadas anteriormente, ou seja, anos de escolaridade $(h)$ e a depreciação efetiva do capital $(\delta+n+g)$ são provenientes de Barro e Lee (2001) e o investimento em capital físico é da Penn World Tables 6.1.

Foram utilizados os métodos $\mathrm{MQO}^{13}$ e de dados de painel para analisar a importância da especificidade de cada país depois que controlamos pelas variáveis da equação (17). Pelo fato de o termo de erro aleatório $(\varepsilon)$ ser específico para cada país, é possível que exista uma correlação entre $\varepsilon$ e pelo menos uma das variáveis independentes da equação (17). Seguindo as palavras de Islam "panel data framework provides a better and more natural setting to control for this technology shift term ع.” (1995, p. 1134-35).

Esse método de estimação é uma forma mais adequada para se analisar preferências e tecnologia entre os países, que são variáveis de difícil mensuração. É importante ressaltar que, pelo fato de essas especificidades não estarem mais nos resíduos, a probabilidade de que estes sejam correlacionados com uma das variáveis explicativas é menor. Adicionalmente, a utilização de variáveis explicativas defasadas como instrumentos elimina esse problema.

Também foram utilizadas as variáveis em logaritmo natural $(\ln )$ para testar a robustez dos resultados, além das variáveis explicativas defasadas para controlar para o problema de endogeneidade. $^{14}$

Utilizando dados de painel, temos que decidir entre efeitos fixos e aleatórios. A estimação por efeitos fixos assume que as diferenças entre as unidades de análise podem ser consideradas como mudanças paramétricas da função de produção. $\mathrm{O}$ método de estimação via efeitos aleatórios tem como suposição que a especificidade de cada unidade de análise é distribuída de forma aleatória. A principal desvantagem desse método é a suposição de que as especificidades de cada unidade de análise capturada por esse método de estimação não são correlacionadas com os outros regressores.

Como a nossa principal motivação para a utilização de dados de painel é justamente pelo fato de que esses efeitos individuais podem estar correlacionados com alguma outra variável independente, efeitos fixos é o método de análise mais apropriado na presente ocasião. Adicionalmente, os testes de Hausman para testar qual o método mais adequado não foram conclusivos devido à violação das condições assintóticas necessárias a sua aplicação.

13 Os resultados não foram apresentados, mas estão disponíveis com os autores.

14 É importante ressaltar que a especificação seria outra quando se utilizam variáveis em logaritmo natural. Essa outra especificação não foi feita, pois iria além dos objetivos do presente artigo. 


\section{Resultados}

\subsection{Resultados estimados utilizando a menor amostra (29 países)}

Na Tabela 1 estão os resultados das regressões estimadas utilizando o modelo da equação (17) para a menor amostra. A variável dependente é a taxa de crescimento da renda por trabalhador nas duas primeiras regressões e logaritmo natural $(\ln )$ da mesma nas duas últimas. O método utilizado na estimação da equação de regressão foi o de Efeitos Fixos (EF), em todos os casos.

$\mathrm{Na}$ primeira coluna estão as variáveis explicativas do modelo. $\mathrm{Na}$ segunda são apresentados os resultados utilizando os dados com a variável explicada e as explicativas no mesmo período de tempo, enquanto que, na terceira, todas as variáveis explicativas estão defasadas em um período (cinco anos), para controlar o efeito de endogeneidade e evitar problemas de tendenciosidade dos estimadores.

Na quarta coluna são apresentados os resultados com todas as variáveis em logaritmo natural. O objetivo desta é testar a robustez dos resultados, além de facilitar a interpretação dos mesmos, já que os coeficientes se tornam elasticidades. No entanto, mais ênfase será dada aos resultados utilizando as variáveis que não estão em logaritmo natural devido à especificação do modelo. Finalmente, na última estão os resultados com todas as variáveis em logaritmo natural e com as variáveis explicativas defasadas. O motivo é o mesmo apresentado anteriormente.

Nas três primeiras estimações, os resultados apresentados estão corrigidos para problemas de autocorrelação, heterocedasticidade e normalidade dos resíduos. O problema da autocorrelação não é grave em nenhuma delas, mas a utilização do método dos Mínimos Quadrados Generalizados Iterativo de Prais-Winsten e Cochrane-Orcutt reduziu o grau de autocorrelação. Na última equação de regressão estimada (última coluna), os resultados apresentados foram corrigidos para problemas de heterocedasticidade e normalidade dos resíduos. Para sanar tal problema, foi utilizado o método Iterativo dos Mínimos Quadrados com Redistribuição de Pesos.

Comparando os resultados das equações estimadas, a primeira coisa a se notar é que o coeficiente da variável de depreciação efetiva do capital $(n)$ não é estatisticamente diferente de zero em nenhum dos casos.

Nas duas primeiras equações estimadas (EF e EF LAG) é interessante notar a troca de sinal do coeficiente do investimento em capital físico por trabalhador ( $i k)$. Nos dois casos, os coeficientes são estatisticamente diferentes de zero. Essa troca também acontece nos resultados apresentados nas duas últimas colunas, apesar de o coeficiente não ser estatisticamente diferente de zero nos resultados da penúltima. Os resultados da quinta coluna indicam que, para $1 \%$ de aumento no investimento de capital por trabalhador, resulta em uma queda de $0,37 \%$ na taxa de crescimento da renda por trabalhador. 
Tabela 1 - Efeitos diretos e indiretos do capital humano sobre a taxa de crescimento da renda (menor amostra)

\begin{tabular}{|c|c|c|c|c|}
\hline (1) & (2) & (3) & (4) & (5) \\
\hline & $\mathrm{EF}$ & EF LAG & EF LN & EF LAG LN \\
\hline \multirow[t]{2}{*}{$n$} & 0.170 & $-9.50 e-3$ & 0.036 & $-9.42 e-3$ \\
\hline & $(0.385)$ & $(8.82 e-3)$ & $(0.024)$ & $(19.85 \mathrm{e}-3)$ \\
\hline \multirow[t]{2}{*}{$i k$} & $1.57 e-05$ & $-3.65 e-05$ & 0.097 & -0.376 \\
\hline & $(5.91 \mathrm{e}-06)^{\star *}$ & $(9.51 \mathrm{e}-06)^{\star *}$ & $(0.051)$ & $(0.068)^{\star *}$ \\
\hline \multirow[t]{2}{*}{$i h$} & -0.0524 & -0.0480 & -0.0869 & -0.073 \\
\hline & $(0.0237)^{*}$ & $(0.0291)$ & $(0.0259)^{\star *}$ & $(0.046)$ \\
\hline \multirow[t]{2}{*}{$s p$} & $-2.27 e-04$ & $4.09 \mathrm{e}-05$ & -0.158 & -0.066 \\
\hline & $(1.49 \mathrm{e}-04)$ & $(1.7 e-04)$ & $(0.0353)^{\star}$ & $(0.076)$ \\
\hline \multirow[t]{2}{*}{$a h$} & 0.706 & 0.644 & 0.287 & -3.892 \\
\hline & $(0.296)^{*}$ & $(0.288)^{*}$ & $(0.108)^{\star \star}$ & (104.63) \\
\hline \multirow[t]{2}{*}{ afdi } & $1.85 e-03$ & 0.0079 & -4.86 & -3.033 \\
\hline & $(1.28 \mathrm{e}-03)$ & $(0.002)^{* *}$ & $(1.16)^{\star *}$ & $(1.53)^{*}$ \\
\hline \multirow[t]{2}{*}{$a m$} & $6.14 \mathrm{e}-08$ & $4.44 \mathrm{e}-08$ & 5.24 & -0.982 \\
\hline & $(1.28 \mathrm{e}-08)^{\star *}$ & $(2.08 \mathrm{e}-08)^{*}$ & $(1.17)^{\star *}$ & (104.09) \\
\hline \multirow[t]{2}{*}{ afdih } & $-1.81 \mathrm{e}-04$ & $-6.37 e-04$ & 4.85 & 3.021 \\
\hline & $(1.26 \mathrm{e}-04)$ & $(2.02 \mathrm{e}-04)^{* *}$ & $(1.15)^{* *}$ & $(1.523)^{*}$ \\
\hline \multirow[t]{2}{*}{$a m h$} & $-1.22 \mathrm{e}-08$ & $-8.92 e-09$ & -5.14 & 1.123 \\
\hline & $(2.51 \mathrm{e}-09)^{* *}$ & $(4.02 \mathrm{e}-09)^{*}$ & $(1.16)^{\star *}$ & (104.10) \\
\hline \multirow[t]{2}{*}{$c$} & -0.66 & 0.0945 & Dropped & -3.640 \\
\hline & $(0.43)$ & $(0.206)$ & & (138.44) \\
\hline$N$ & 112 & 84 & 102 & 76 \\
\hline$R^{2}$ & 0.87 & 0.95 & 0.92 & 0.80 \\
\hline
\end{tabular}

Notas: o desvio padrão de cada estimador se encontra entre parênteses. * significante ao nível de 5\%; ** significante ao nível de 1\%. A variável dependente é a taxa de crescimento da renda por trabalhador (equações 1 e 2) e o logaritmo natural da mesma (equações 3 e 4), $n$ é a taxa de crescimento de trabalhadores de cada país, ih é a variação de anos de escola das pessoas com 25 anos ou mais. A variável $i k$ é o investimento em capital físico por trabalhador, $s p$ é o número de publicações científicas por habitante, ah é $\left[\left(T-A_{i}\right) / A_{i}\right]$ multiplicado por $h$, onde $T$ é o nível de tecnologia na fronteira e $A_{i}$ é o nível de tecnologia do país $i$, afdi é o termo de interação entre $\left[\left(T-A_{i}\right) / A_{i}\right]$ e $I E D$. A variável am é o termo de interação entre importações e a distância da fronteira, afdih é o termo de interação entre afdi e $h, a m h$ é a interação entre am e $h$, c é a constante, $N$ é o tamanho da amostra e $R^{2}$ é o coeficiente de determinação.

Devido ao tempo de maturação dos investimentos em capital físico e, portanto, do lag existente entre a sua realização e a geração de capacidade produtiva, além do controle da relação de bicausalidade entre essas variáveis ao se empregar variáveis defasadas, os resultados apresentados nas colunas (2) e (4) são mais confiáveis. Desse modo, controlando para as demais variáveis do modelo, o investimento em capital físico parece ter um impacto negativo sobre a taxa de crescimento econômico.

O investimento em capital humano $(i h)$, que parecia ter um impacto negativo na primeira equação, perde significância quando se consideram as equações com variáveis defasadas. O mesmo 
acontece com as estimações empregando as variáveis em logaritmo natural. Seguindo a mesma linha de raciocínio do parágrafo anterior, a conclusão é que essa variável não afeta a taxa de crescimento econômico quando se controla para as demais variáveis utilizadas na especificação da equação (17). Esses resultados são semelhantes aos encontrados por outros autores, como Romer (1990), Benhabib e Spiegel (1994), Hall e Jones (1998) e Pritchett (2001).

Ao contrário das conclusões de alguns modelos de crescimento endógeno, em que o motor do crescimento é a quantidade de recursos alocados em P\&D, como Romer (1990), Grossman e Helpman (1991a,1991b) e Aghion e Howitt (1992), os coeficientes das proxies para essa variável (sp) são negativos em todos os casos e significativos, ao nível de 5\%, na regressão de EF LN. Os resultados da coluna (4) ainda indicam que uma elevação de $1 \%$ no número de publicações científicas por habitante (sp) reduz a taxa de crescimento em $0,16 \%$ para esse grupo de países.

Apesar de a proxy para inovação não ser significativa, o capital humano se mostra relevante no processo de difusão de tecnologia, pois, quanto maior a quantidade de capital, mais relevante é o efeito positivo sobre a taxa de crescimento de se estar longe da fronteira tecnológica. Esse efeito é capturado pela variável de interação entre o capital humano e a fronteira $(a h)$, cujos coeficientes são positivos e significativos nas três primeiras estimações.

Os níveis de importações e investimento estrangeiro direto também ajudam no processo de difusão, pois quanto maior o nível dessas duas variáveis, mais importante é o efeito da distância da fronteira tecnológica. Isso pode ser visto pelos coeficientes positivos e significativos do termo de interação entre essas variáveis e a fronteira tecnológica (afdi e am) nas duas primeiras equações. Nas últimas equações os coeficientes e afdi são negativos e significativos, enquanto que o de $a m$ é positivo e significativo na terceira equação.

Como dito anteriormente, a preferência é dada aos resultados apresentados na coluna 3, devido à especificação do modelo e à defasagem das variáveis explicativas. Portanto, conclui-se que os efeitos dessas variáveis sobre o crescimento são positivos.

Finalmente, os coeficientes da interação destas com o nível de capital humano possuem sinais negativos e são significativos nas duas primeiras equações, exceto o coeficiente de afdih na primeira regressão. Nas duas outras, os coeficientes de afdih são positivos e significativos, enquanto que o coeficiente de amh continua negativo e significativo nos resultados apresentados na quarta coluna.

Desse modo, a conclusão que se chega ao analisar os resultados da Tabela 1 é que o papel do capital humano parece ser mais importante via difusão de tecnologia, mesmo para os países ricos, pois os coeficientes de interação entre capital humano e distância da fronteira $(a h)$ são positivos em todos os casos e significativos, exceto para os resultados apresentados na coluna (5).

Investimento Estrangeiro Direto (IED) também parece ser importante no processo de difusão e, portanto, sobre a taxa de crescimento da renda por trabalhador. Os coeficientes de afdi são positivos e significativos nos resultados das colunas (2) e (3). No entanto, a interação dessa variável com o capital humano (afdih) é negativa. Esse resultado parece ser contra-intuitivo, pois indica que, apesar do IED ser um importante canal de difusão, ele perde importância à medida que aumenta o nível de capital humano. 
Os resultados também indicam que as importações $(\mathrm{am})$ são relevantes no processo de difusão. Os coeficientes da interação do capital humano com essa variável $(A m h)$ têm sinais negativos e significativos em quase todos os casos, contrariando os resultados encontrados por Coe, Helpman e Hoffmaister (1997).

\subsection{Resultados estimados utilizando a maior amostra (96 países)}

A descrição da Tabela 2 é semelhante à da Tabela 1. A diferença é que a amostra utilizada para análise é a maior, composta por 96 países. Comparando os resultados das quatro estimações, os resultados são mais robustos que os da Tabela 1, provavelmente devido ao maior tamanho da amostra.

As estimações foram corrigidas para problemas de heterocedasticidade e normalidade dos resíduos. Para sanar tal problema, foi utilizado o método Iterativo dos Mínimos Quadrados com Redistribuição de Pesos. Em nenhum dos casos ocorreu o problema de heterocedasticidade.

Novamente, os coeficientes da depreciação efetiva do capital $(n)$ não são significativos em nenhum dos casos e o padrão de mudança da variável investimento em capital físico é exatamente o mesmo da Tabela 1. Ou seja, quando se considera o seu efeito defasado, ele é negativo. De acordo com o coeficiente estimado na última coluna, para cada $10 \%$ a mais de investimento em capital físico, a taxa de crescimento se reduz em $0,72 \%$, em média. Esse efeito é consideravelmente menor do que aquele apresentado na Tabela 1, indicando que o capital físico tem uma maior importância para os países com menor nível de renda. Seria interessante testar, em estudo futuros, se o efeito desse fator sobre a taxa de crescimento é positivo quando se excluem os países da menor amostra.

Os efeitos diretos do capital humano são negativos quando se consideram as variáveis no mesmo período de tempo, mas perdem a significância com as variáveis defasadas, assim como nos resultados apresentados na Tabela 1 . Causa estranheza a magnitude dos coeficientes, mas considerando que não são significativos com variáveis defasadas, não há benefícios em realizar uma análise mais detalhada nesse ponto.

Novamente, de forma similar aos resultados apresentados na Tabela 1, o papel do capital humano parece não ser relevante para estimular a economia via inovação. Esse resultado é mais coerente nesse caso, pois o processo de criação de tecnologia não é relevante na grande maioria dos países que compõem essa amostra. 
Tabela 2 - Efeitos diretos e indiretos do capital humano sobre a taxa de crescimento da renda (maior amostra)

\begin{tabular}{|c|c|c|c|c|}
\hline (1) & (2) & (3) & (4) & (5) \\
\hline & $E F$ & EF LAG & EF LN & EF LAG LN \\
\hline \multirow[t]{2}{*}{$n$} & 0.138 & -0.0128 & -1.11 & -0.021 \\
\hline & $(0.219)$ & $(0.0376)$ & $(0.25)$ & $(0.079)$ \\
\hline \multirow[t]{2}{*}{$i k$} & $3.14 \mathrm{e}-05$ & $-4.2 \mathrm{e}-05$ & 0.084 & -0.072 \\
\hline & $(5.62 \mathrm{e}-06)^{* *}$ & $(7.32 \mathrm{e}-06)^{\star *}$ & $(0.024)^{\star *}$ & $(0.025)^{\star *}$ \\
\hline \multirow[t]{2}{*}{$i h$} & -367 & 16.96 & -285.65 & -44.97 \\
\hline & $(116.54)^{\star *}$ & $(148.56)$ & $(100.09)^{* *}$ & $(118.80)$ \\
\hline \multirow[t]{2}{*}{$p$} & $-5.66 e-06$ & $-1.45 e-06$ & -0.034 & 0.011 \\
\hline & $(2.47 e-06)^{*}$ & $(3.78 \mathrm{e}-06)$ & $(0.0202)$ & $(0.025)$ \\
\hline \multirow[t]{2}{*}{$a h$} & $1.37 e-03$ & $3.11 \mathrm{e}-03$ & -0.119 & 0.532 \\
\hline & $(8.76 e-04)$ & $(1.13 e-03)^{*}$ & $(0.0698)$ & $(0.079)^{* *}$ \\
\hline \multirow[t]{2}{*}{ afdi } & $4.73 e-05$ & $3.39 e-05$ & -0.061 & -0.209 \\
\hline & $(2.31 e-05)^{*}$ & $(3.61 e-05)$ & $(0.044)$ & $(0.057)^{* *}$ \\
\hline \multirow[t]{2}{*}{$a m$} & $2.24 \mathrm{e}-10$ & $1.06 \mathrm{e}-09$ & -0.060 & -0.121 \\
\hline & $(1.62 \mathrm{e}-10)$ & $(4.37 \mathrm{e}-10)^{*}$ & $(0.058)$ & $(0.0678)$ \\
\hline \multirow[t]{2}{*}{ afdih } & $-5.42 \mathrm{e}-06$ & $6.45 \mathrm{e}-06$ & 0.049 & 0.095 \\
\hline & $(2.55 \mathrm{e}-06)^{*}$ & $(4.68 \mathrm{e}-06)$ & $(0.023)^{*}$ & $(0.025)^{* *}$ \\
\hline \multirow[t]{2}{*}{$a m h$} & $-4.81 e-11$ & $-1.84 e-10$ & 0.103 & -0.087 \\
\hline & $(3.07 e-11)$ & $(7.35 \mathrm{e}-11)^{*}$ & $(0.078)$ & $(0.088)$ \\
\hline \multirow[t]{2}{*}{$c$} & -0.99 & 0.222 & -0.884 & 3.383 \\
\hline & $(0.59)$ & $(0.225)$ & $(0.338)^{* *}$ & $(0.399)$ \\
\hline$N$ & 366 & 272 & 357 & 266 \\
\hline$R^{2}$ & 0.65 & 0.73 & 0.64 & 0.82 \\
\hline
\end{tabular}

Notas: o desvio padrão de cada estimador se encontra entre parênteses. * significante ao nível de 5\%; ** significante ao nível de $1 \%$. A variável dependente é a taxa de crescimento da renda por trabalhador (equações 1 e 2) e o logaritmo natural da mesma (equações 3 e 4), $n$ é a taxa de crescimento de trabalhadores de cada país, ih é a variação de anos de escola das pessoas com 25 anos ou mais. A variável $i k$ é o investimento em capital físico por trabalhador, $p$ é o número de patentes por habitante concedidas, $a h$ é $\left[\left(T-A_{i}\right) / A_{i}\right]$ multiplicado por $h$, onde $T$ é o nível de tecnologia na fronteira e $A_{i}$ é o nível de tecnologia do país $i$, afdi é o termo de interação entre $\left[\left(T-A_{i}\right) / A_{i}\right]$ e IED. A variável am é o termo de interação entre importações e a distância da fronteira, afdih é o termo de interação entre afdi e $h$, amh é a interação entre $a m$ e $h, c$ é a constante, $N$ é o tamanho da amostra e $R^{2}$ é o coeficiente de determinação.

Outra vez, o impacto do capital humano parece ser mais importante via difusão, visto que o coeficiente da variável de interação entre capital humano e a distância da fronteira $(a h)$ é positivo e significativo quando se empregam variáveis defasadas. Pela magnitude do coeficiente na última coluna, esse impacto é assaz relevante: para cada $1 \%$ de elevação na distância da fronteira tecnológica, a taxa de crescimento da renda por trabalhador se eleva em $0,53 \%$. Adicionalmente, ao serem comparados os coeficientes dessa variável nas colunas 2 e 3 das duas tabelas, nota-se que o efeito do capital humano sobre a difusão é consideravelmente maior quando se considera a maior amostra.

O efeito dos investimentos estrangeiros diretos parece não ser significativo. Considerando as variáveis defasadas em $l n$, talvez até seja negativo. Esse resultado é o contrário do esperado, pois os 
resultados da Tabela 1 mostraram que esse efeito é positivo para os países mais avançados e, como ressaltado por Blomström e Kokko (1998), espera-se que o efeitos do IED sejam mais importantes para os países em desenvolvimento, visto que firmas multinacionais complementam o deficiente sistema de formação de capital humano das economias menos desenvolvidas.

No entanto, sua interação com a variável capital humano parece ser positiva, de acordo com o coeficiente de afdih, na última coluna. Um conclusão que se pode tirar é que, quando se considera uma ampla gama de países, aqueles que têm um maior nível de capital humano são os que conseguem tirar um maior proveito desse processo, o que está de acordo com os resultados de Borensztein et al. (1998), Xu (2000) e Dimelis e Louri (2003).

Já a interação das importações com a distância da fronteira tem um efeito positivo ao auxiliar a difusão de tecnologia, considerando os resultados da coluna 3. No entanto, quando se considera sua interação com o nível de capital humano, o resultado é negativo, como na Tabela 1, ou seja, novamente contraria os resultados de Coe, Helpman e Hoffmaister (1997).

De acordo com os resultados apresentados, novamente se chega à conclusão de que o principal papel do capital humano sobre a taxa de crescimento econômico é por meio do seu estímulo à difusão de tecnologia. Seus impactos diretos, ou seja, na melhora das habilidades dos trabalhadores e na criação de tecnologia parecem não ser relevantes.

Outro ponto que merece ser destacado é o papel das importações sobre o crescimento por meio da maior difusão de tecnologia. Isso acontece porque o comércio internacional disponibiliza bens que incorporam conhecimento externo, fornecendo tecnologia que, de outro modo, não estaria disponível ou que seria muito mais custosa para ser obtida.

\section{CONCLUSÕES}

A introdução das várias formas em que o capital humano influencia a taxa de crescimento da renda por trabalhador tem como objetivo a utilização de um modelo mais completo para evitar possíveis erros de especificação, pois, no caso de omissão de uma variável relevante, que esteja correlacionada com pelo menos uma variável explicativa, obteríamos coeficientes tendenciosos e inconsistentes (os que têm correlação com a variável omitida).

Por exemplo, no modelo utilizado no presente estudo, a omissão dos canais indiretos, em que o capital humano afeta a taxa de crescimento da renda por trabalhador, elevaria seus efeitos diretos, caso ele seja correlacionado positivamente com as variáveis omitidas. Assim, poderíamos concluir que seu papel direto é positivo e significante, como encontrado em alguns estudos que só levam em conta esse canal, por exemplo, Mankiw, Romer e Weil (1992). No entanto, pelos resultados obtidos, concluímos que seu efeito direto sobre o crescimento da renda por trabalhador não é relevante em nenhuma das amostras, considerando as regressões com variáveis defasadas.

O padrão de mudança do efeito da variável investimento por trabalhador sobre a variável explicada é intrigante e precisa de uma análise mais detalhada. No entanto, controlando para as várias formas em que o capital humano afeta o crescimento e considerando as regressões em que 
esse fator entra com um período de 5 anos de defasagem, ele parece ter um papel negativo sobre a taxa de crescimento, sobretudo para os países mais desenvolvidos.

Outro resultado contrário ao esperado é a não-significância dos coeficientes das proxies para gastos em $\mathrm{P} \& \mathrm{D}$, ou seja, os resultados desse processo (publicações científicas e patentes registradas). Uma possível explicação seria que os países engajados na criação de tecnologia são os que estão na fronteira e, pelo próprio processo de convergência, são os que menos crescem. Em futuros estudos, seria importante desenvolver um modelo que incluísse o nível da renda por trabalhador no começo do período para contornar esse problema, embora Jones (1995a, 1995b, 2002) encontre evidências empíricas de que não há relação entre a quantidade de capital humano destinada à criação de tecnologia e taxa de crescimento.

Como enfatizado por Nelson e Phelps (1966) e de acordo com os resultados de outros estudos, como Benhabib e Spiegel (1994, 2002) e Islam (1995), o capital humano parece ser mais importante pela sua interação com a distância da fronteira, ou seja, pela aceleração do processo de difusão tecnológica, principalmente nos países de renda mais elevada. Os resultados do presente estudo dão suporte a tal idéia, visto que os coeficientes de interação entre capital humano e a distância da fronteira tecnológica são positivos e significativos para as duas amostras. Assim, o processo de difusão também é relevante para os países mais desenvolvidos.

Segundo a teoria econômica, o nível de Investimento Estrangeiro Direto (IED) tem um papel importante na difusão. De acordo com os resultados empíricos, o seu nível parece ser um importante canal de difusão para os países desenvolvidos, mas não para aqueles em desenvolvimento.

As importações também parecem ser relevantes no processo de difusão nas duas amostras, ou seja, tanto para o grupo de países desenvolvidos quanto para aqueles em desenvolvimento. Os efeitos das importações estão de acordo com outros estudos como Coe e Helpman (1995) e Coe, Helpman e Hoffmaister (1997). No entanto, de acordo com o efeito de interação dessa variável com o nível de capital humano, os países que possuem um maior nível de capital humano parecem tirar menor proveito das oportunidades de difusão e spillovers geradas pelas importações.

O mais importante resultado do presente estudo é o fato de o principal efeito do capital humano ser sobre a difusão de tecnologia, tanto para o grupo de países desenvolvidos quanto para o grupo em desenvolvimento. Assim, entre o modelo de Lucas (1988) e o de Nelson e Phelps (1996), a opção seria pelo segundo. Adicionalmente, esse resultado ajuda a explicar a falta de significância do efeito do capital humano sobre o crescimento em alguns estudos empíricos quando se considera apenas seu efeito direto.

\section{REFERÊNCIAS BIBLIOGRÁFICAS}

AGHION, P. ; HOWITT, P. A model of growth through creative destruction. Econometrica, v. 60, n. 2, p. 323-351, 1992.

BARRO, R. J.; LEE, J. W. International data on educational attainment: update and implications. Oxford Economic Papers, v. 53, n. 3, p. 541-563, 2001. 
BECKER, G. S. Human capital: a theoretical and empirical analysis with special reference to education. 3. ed. Chicago: The University of Chicago, 1993. p. 3-130.

BECKER, G. S. Investment in human capital: a theoretical analysis. The Journal of Political Economy, v. 70, n. 5, p. 9-49, 1962.

BENHABIB, J.; SPIEGEL, M. M. Human capital and technology diffusion. New York, NY: Federal Reserve Bank of San Francisco, 2002. (Working paper; 2003-02). Disponível em: <http://www.frbsf. org/publications/economics/papers/2003/wp03-02bk.pdf>.

BENHABIB, J.; SPIEGEL, M. M. The role of human capital in economic development: evidence from aggregate cross-country data. Journal of Monetary Economics, v. 34, n. 2, p. 143-173, 1994.

BLOMSTRÖM, M.; KOKKO, A. Multinational corporations and spillovers. Journal of Economic Surveys, v. 12, n. 2, p. 1-31, 1998.

BORENSZTEIN, E.; DE GREGORIO, J.; LEE, J. W. How does foreign direct investment affect economic growth. Journal of International Economics, v. 45, n. 1, p. 115-135, June 1998.

COE, D. T.; HELPMAN, E. International R\&D spillovers. European Economic Review, v. 39, n. 5, p. 859-887, 1995.

COE, D. T.; HELPMAN, E.; HOFFMAISTER, A. W. North-South R\&D spillovers. The Economic Journal, v. 107, n. 440, p. 134-149, 1997.

CONNOLLY, M. P. Human capital and growth in the Post-Bellum South: a separate but unequal story. The Journal of Economic History, v. 64, n. 2, p. 1-39, 2004.

. The dual nature of trade: measuring its impact on imitation and growth. Journal of Development Economics, v. 72, n. 1, p. 31-55, 2003.

DIMELIS, S.; LOURI, H. Foreign direct investment and technology spillovers: which firms really benefit? Athens: Athens University of Economics and Business, Laboratory of Economic Policy Studies, 2003. $28 \mathrm{p}$.

DOWRICK, S. Ideas and education: level or growth effects? Cambridge, MA: National Bureau of Economic Research, 2003.30p. (Working paper, 9709)

GROSSMAN, G. M.; HELPMAN, E. Quality ladders and product cycles. The Quarterly Journal of Economics, v. 106, n. 2, p. 557-586, 1991a. $1991 \mathrm{~b}$

. Quality ladders in the theory of growth. The Review of Economic Studies, v. 58, n. 1, p. 43-61,

HALL, R. E.; JONES, C. I. Why some countries produce so much more output per worker than others? Cambridge, MA.: National Bureau of Economic Research, 1998. 49p. (Working Paper, 6564)

HANUSHEK, E. A.; KIMKO, D. D. Schooling, labor-force quality, and the growth of nations. The American Economic Review, v. 90, n. 5, p. 1184-1208, 2000.

ISLAM, N. Growth empirics: a panel data approach. The Quarterly Journal of Economics, v. 110, n. 4, p. 1127-1170, 1995.

JAVORCIK, B. S. Does foreign direct investment increase the productivity of domestic firms? In search of spillovers through backward linkages. The American Economic Review, v. 94, n. 3, p. 605-627, June 2004.

JONES, C. I. Sources of U.S. economic growth in a world of ideas. The American Economic Review, v. 92, n. 1, p. 220-239, 2002.

. R\&D based models of economic growth. The Journal of Political Economy, v. 103, n. 4, p. 759784, 1995a. 
. Time series tests of endogenous growth models. The Quarterly Journal of Economics, v. 110, n. 2, p. 495-525, 1995b.

KELLER, W. International technology diffusion. Journal of Economic Literature, v. 42, n. 3, p. 752-782, Sept. 2004.

. How trade patterns and technology flows affect productivity growth. Cambridge, MA.: National Bureau of Economic Research, 1999. 55p. (Working paper, 6990).

LUCAS, R. E. Jr. On the mechanics of economic development. Journal of Monetary Economics, v. 22, n. 1, p. 3-42, 1988.

MANKIW, N. G.; ROMER, D.; WEIL, D. A contribution to the empirics of economic growth. The Quarterly Journal of Economics, v. 107, n. 2, p. 407- 437, 1992.

NAKABASHI, L. Três ensaios sobre capital humano e renda por trabalhador. Tese (Doutorado) - CEDEPLAR/UFMG, Belo Horizonte, 2005.

NELSON, R. R.; PHELPS, E. S. Investment in humans, technological diffusion, and economic growth. The American Economic Review, v. 56, n. 2, p. 69-75, 1966.

OECD science, technology and industrial outlook. Paris: Organization for Economic Co-operation and Development, 2002.328p.

PRITCHETT, L. Where has all the education gone? The World Bank Economic Review, v. 15, n. 3, p. 367-391, 2001.

ROMER, P. Endogenous technological change. The Journal of Political Economy, v. 98, n. 5, p. 71-102, 1990. $10 \overline{3} \overline{7}, 1986$.

SCHULTZ, T. W. Capital formation by education. The Journal of Political Economy, v. 68, n. 6, p. 571583, 1960.

. Investment in human capital. The American Economic Review, v. 51, n. 1, p. 1-17, 1961.

. Reflections on investment in man. The Journal of Political Economy, v. 70, n. 5, p. 1-8, 1962.

SOLOW, R. M. A contribution to the theory of economic growth. The Quarterly Journal of Economics, v. 70, n. 1, p. 65-94, 1956.

TELES, K. V. The role of human capital in economic growth. Applied Economic Letters, v. 12, n. 9, p. 583-587, 2005.

TEMPLE, J. R. W. A positive effect of human capital on growth. Economic Letters, v. 65, n. 1, p. 131-134, 1999.

XU, B. Multinational enterprises, technology diffusion, and host country productivity growth. Journal of Development Economics, v. 62, n. 2, p. 477-493, Aug. 2000. 


\section{APÊNDICE A - PaísES QUe COMPÕEM AS DUAS AMOSTRAS}

Quadro A1 - Países que compõem a menor amostra

\begin{tabular}{|l|l|l|c|}
\hline Austrália & Alemanha Unificada & Japão & Portugal \\
Áustria & Grécia & Coréia & Espanha \\
Bélgica & Hungria & México & Suécia \\
Canadá & Islândia & Holanda & Turquia \\
China & Irlanda & Nova Zelândia & Reino Unido \\
Dinamarca & Israel & Noruega & Estados Unidos \\
Finlândia & Itália & Polônia & \\
França & & & \\
\hline
\end{tabular}

Quadro A2 - Países que compõem a maior amostra

\begin{tabular}{|c|c|c|c|c|}
\hline Argélia & Dinamarca & Índia & Holanda & Espanha \\
\hline Argentina & Rep. Dominicana & Indonésia & Nova Zelândia & Sri Lanka \\
\hline Austrália & Equador & Iran & Nicarágua & Suécia \\
\hline Áustria & Egito & Irlanda & Nigéria & Suíça \\
\hline Bangladesh & El Salvador & Israel & Noruega & Síria \\
\hline Barbados & Fiji & Itália & Paquistão & Taiwan \\
\hline Bélgica & Finlândia & Jamaica & Panamá & Tailândia \\
\hline Benin & França & Japão & Nova Guiné & Togo \\
\hline Bolívia & Gâmbia & Jordan & Paraguai & Trindad e Tob. \\
\hline Botswana & Alemanha Unificada & Kenya & Peru & Tunísia \\
\hline Brasil & Gana & Coréia & Filipinas & Turquia \\
\hline Camarão & Grécia & Lesotho & Polônia & Uganda \\
\hline Canadá & Guatemala & Malawi & Portugal & Reino Unido \\
\hline África Central & Guiana & Malásia & Romênia & Estados Unidos \\
\hline Chile & Haiti & Mali & Ruanda & Uruguai \\
\hline China & Honduras & Mauritius & Senegal & Venezuela \\
\hline Colômbia & Hong Kong & México & Serra Leão & Zaire \\
\hline Congo & Hungria & Moçambique & Cingapura & Zâmbia \\
\hline $\begin{array}{l}\text { Costa Rica } \\
\text { Chipre }\end{array}$ & Islândia & Nepal & África do Sul & Zimbábue \\
\hline
\end{tabular}




\section{APÊNDICE B - ESTATÍSTICAS DESCRITIVAS}

\section{Tabela B1 - Menor amostra}

\begin{tabular}{lcrrrr}
\hline Variáveis & Observações & \multicolumn{1}{c}{ Média } & Desvio Padrão & Mínimo & Máximo \\
\hline$d y$ & 114 & 0.11 & 0.11 & -0.10 & 0.58 \\
$n$ & 116 & 0.11 & 0.38 & -0.02 & 4.17 \\
$i k$ & 115 & 8717.97 & 3518.60 & 497.66 & 16201.21 \\
$i h$ & 116 & 0.35 & 0.40 & -0.61 & 2.45 \\
$h$ & 116 & 8.60 & 2.05 & 3.38 & 12.25 \\
$a h$ & 116 & 0.53 & 0.38 & 0 & 1.5642 \\
$s p$ & 114 & 425.38 & 285.15 & 4.00 & 1019.00 \\
$f d i$ & 116 & 889.64 & 1930.43 & -159.61 & 12312.85 \\
$m$ & 116 & $4.65 \mathrm{E}+06$ & $2.27 \mathrm{E}+07$ & 981.77 & $1.85 \mathrm{E}+08$ \\
$a f d i$ & 116 & 39.36 & 72.61 & -11.63 & 478.12 \\
$a m$ & 116 & $1.50 \mathrm{E}+06$ & $7.41 \mathrm{E}+06$ & 0.00 & $6.34 \mathrm{E}+07$ \\
$a f d i h$ & 116 & 387.95 & 727.22 & -97.66 & 4824.20 \\
$a m h$ & 116 & $7.95 \mathrm{E}+06$ & $3.54 \mathrm{E}+07$ & 0.00 & $3.05 \mathrm{E}+08$ \\
$T A$ & 116 & 0.06 & 0.05 & 0.00 & 0.27 \\
\hline
\end{tabular}

Tabela B2 - Maior amostra

\begin{tabular}{lcrrrr}
\hline Variáveis & Observações & \multicolumn{1}{c}{ Média } & Desvio Padrão & \multicolumn{1}{c}{ Mínimo } & \multicolumn{1}{c}{ Máximo } \\
\hline$d y$ & 367 & 0.069 & 0.19 & -0.44 & 1.71 \\
$n$ & 384 & 0.153 & 0.32 & -0.02 & 4.88 \\
$i k$ & 369 & 3875.959 & 4285 & 0.45 & 16825 \\
$i h$ & 368 & 0.000 & 0.00009 & 0.00 & 0.00 \\
$h$ & 384 & 5.667147 & 2.92 & 0.42 & 12.25 \\
$a h$ & 384 & 40.31935 & 67.47405 & 0 & 640.7611 \\
$p$ & 384 & 1304.965 & $7.71 \mathrm{E}+03$ & 0 & 95139.55 \\
$f d i$ & 384 & $8.52 \mathrm{E}+07$ & $1.67 \mathrm{E}+09$ & -4550 & $3.27 \mathrm{E}+10$ \\
$m$ & 376 & $4.54 \mathrm{E}+13$ & $3.93 \mathrm{E}+14$ & 449.05 & $6.22 \mathrm{E}+15$ \\
$a f d i$ & 384 & $9.54 \mathrm{E}+07$ & $1.87 \mathrm{E}+09$ & -941.78 & $3.66 \mathrm{E}+10$ \\
$a m$ & 376 & $8.26 \mathrm{E}+07$ & $6.31 \mathrm{E}+08$ & 0 & $8.69 \mathrm{E}+09$ \\
$a f d i h$ & 384 & $2.38 \mathrm{E}+08$ & $4.66 \mathrm{E}+09$ & -4435.80 & $9.13 \mathrm{E}+10$ \\
$a m h$ & 376 & $3.24 \mathrm{E}+08$ & $2.74 \mathrm{E}+09$ & 0 & $4.17 \mathrm{E}+10$ \\
$T A$ & 384 & 11.89 & 23.91 & 0 & 201.50 \\
\hline
\end{tabular}




\section{ANEXo A - Lista dos SímbOlos DAS VARIÁVEIS UTILIZADAS}

afdi termo de interação entre a distância da tecnologia do país $i$ em relação ao nível de tecnologia da fronteira tecnológica (em termos proporcionais) multiplicado pelo IED por trabalhador

afdih afdi multiplicado multiplicado por $h$.

ah distância da tecnologia do país $i$ em relação ao nível de tecnologia da fronteira tecnológica (em termos proporcionais) multiplicado por $h$.

am termo de interação entre importações por trabalhador e a distância da fronteira.

$a m h$ termo de interação entre $a m$ e $h$.

dy é a taxa de crescimento da renda por trabalhador.

fdi valor dos investimentos estrangeiros diretos em milhões de dólares a preços constantes.

$h \quad$ anos de estudo da população acima de 25 anos.

ih variação de anos de escola das pessoas com 25 anos ou mais.

ik investimento em capital físico.

$m \quad$ valor das importações em milhares de dólares a preços constantes.

$n$ taxa de crescimento da população acima de 25 anos.

$p \quad$ quantidade de patentes concedidas por habitante.

$s p \quad$ publicações científicas por habitante.

TA distância da fronteira tecnológica. 